\title{
THERMODYNAMIC ASSESSMENT OF HUMAN-THERMAL ENVIRONMENT INTERACTION
}

\author{
S. BOREGOWDA, R. HANDY \& W. HUTZEL \\ Department of Mechanical Engineering Technology, Purdue University, West Lafayette, IN, USA.
}

\begin{abstract}
A second law based thermodynamic model to assess the human-thermal environment interaction and measure occupant thermal comfort is developed. It is based on the second-law analysis of an open system in which human elements are combined with the environmental variables to form a holistic model of humanenvironmental interaction. An objective thermal comfort index (OTCI) that combines air temperature, radiant (surface) temperature, relative humidity, metabolic activity, clothing, air velocity, and skin or body temperature is developed to provide an objective measure of thermal comfort. The human thermal responses due to different ambient conditions are obtained using data from an earlier experimental study. The advantage of using an index to quantify thermal comfort is that it can be integrated into an environmental control system to reduce the number of variables that occupants would need to control their personal spaces by providing a smart technology that senses the overall occupant thermal comfort. The OTCI methodology could significantly contribute to the design of occupant-centered energy-efficient sustainable indoor environments.

Keywords: entropy, objective thermal comfort index, second law, thermodynamics.
\end{abstract}

\section{INTRODUCTION}

During the past five decades, numerous studies have been conducted to understand and examine the thermal aspects of the environmental condition and its effects on human thermal comfort. With regard to academic interest, it poses some challenging problems that require the expertise of researchers from different fields such as engineering, psychology, physiology, and medicine. In many cases, one specialist does not know what the other specialist is doing. The need to bridge this gap among the academic disciplines is one of the motivating factors behind this work. Human thermal comfort is a strong function of both psychological and physiological factors. Thermal comfort is influenced by environmental and physical factors such as air temperature, humidity, air movement, physical activity, and clothing. According to the ASHRAE standard [1], human thermal comfort is defined as 'that condition of mind which expresses satisfaction with the thermal environment'. However, there is no accurate method to quantitatively determine the effect of thermal environmental conditions on human thermal comfort. Therefore, there is a strong need to develop an objective measure that would quantitatively provide an indication of thermal comfort in the form of a single number.

A thermal comfort index is traditionally defined as one that combines two or more parameters, such as air temperature, mean radiant temperature, humidity, and air velocity into a single variable [1]. Although many stress indices have been developed, none of them have been found to be satisfactory by Talty [2]. Some of the widely used thermal stress indices are: effective temperature, heat stress index, wet-bulb globe temperature, wind chill index, and predicted mean vote (PMV). It can be concluded that all of the previous studies that have led to the development of thermal comfort indices were either statistically based or at most used the energy balance principle of first law of thermodynamics. However, Aoki $[3,4]$ was the first to introduce the concept of entropy in human thermal physiology. In these studies, he calculated the entropy production in the human body under basal conditions and during exercise but he did not address the issue of thermal comfort. A study conducted by Boregowda [5] involves development of an objective thermal comfort index (OTCI), a global measure of thermal comfort from a thermodynamic standpoint. In order to validate OTCI, human 
thermal responses were obtained by conducting a series of computer simulations using a human thermal model developed by Smith [6] and Fu [7] under different conditions. This computer model is validated by Boregowda and Tiwari [8] and at the National Renewable Energy Laboratory (NREL) by McGuffin et al. [9]. The OTCI was validated against the regression equation for PMV obtained from a large-scale experimental study [1].

\section{MODEL DEVELOPMENT}

\subsection{Physical model}

This unique thermal comfort measurement concept shown in Fig. 1 senses human thermal comfort and activates the environmental control systems to automatically provide the desired comfort for the occupants in the buildings, automobiles, or any space enclosures. This concept combines human peripheral physiological responses, such as skin temperature, and environmental variables, such as the air temperature, relative humidity, and air velocity, to provide an index value of the quality of human-thermal environment interaction. This index value could be used for manual and/or automatic control of occupant environments.

\subsection{Thermal comfort definition}

In this study, entropy generation in the combined human-thermal environment that involves both thermal environmental variables and human thermal responses is being postulated as a quantitative measure

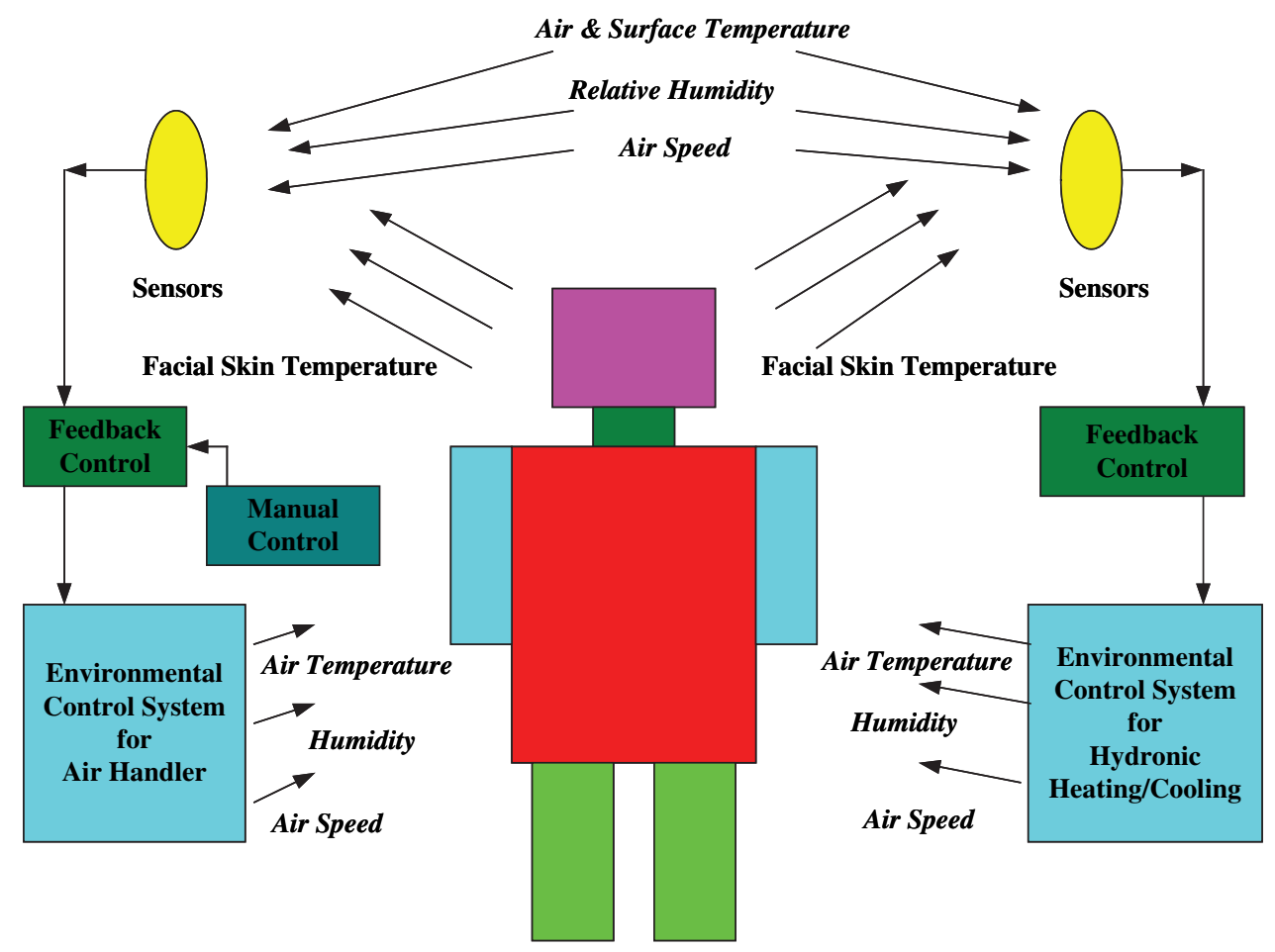

Figure 1: Thermal comfort measurement and control system. 
of thermal comfort accordingly. The ASHRAE [1] definition of thermal comfort is extended to include physiological reaction to changes in the thermal environment and is defined under postulate I as follows:

Postulate I. Thermal comfort is defined as a condition of mind that expresses satisfaction with the thermal environment and is reflected in physiological reaction to corresponding changes in the thermal environment except in cases of individuals with rare diseases and those who have achieved voluntary self-regulation of their physiological functioning.

From the laws of psychophysics, the following logic is established according to the works of famous German Physicist Gustav Fechner [10], who defined the relationship between human mind and its response to physical sensations as:

$$
\text { Psych }=f\{\text { Physical }\}
$$

According to the ASHRAE standard developed by Fanger [11], thermal comfort is a condition of mind that leads us to the understanding that thermal comfort occurs in psychological domain. Thus,

$$
\text { Thermal comfort }=\text { psych }=f\{\text { Physical }\}
$$

The physical system is a combination of human body and thermal environment. Thus, thermal comfort is defined using a thermodynamic function in the following manner.

Thermal comfort $=H \times f\{$ Physiological responses, Thermal environmental variables $\}$

where $H$ is the non-dimensional human coefficient that takes into account the variation in individual responses to thermal environment. It depends on age, sex, race, and other related factors. The value of $H$ was taken to be equal to 1.0 with the assumption of a 'standard human' in the present computer simulation study. However, in experimental studies of the future, the researcher will have to make a decision to assign the suitable value based on acclimatization levels of human subjects. For example, the value of $H$ would be different in tropical and warmer regions of the world where people are used to working in hot and humid climatic conditions. For example, Europeans living in Africa for a long-time get used to the hot climates and respond differently from their counterparts in Europe. The coefficient ' $H$ ' provides a link between physiology and behavior. Although, we might be composed of the same anatomy, why do we respond to the same thermal conditions differently? How do we infer behavior from physiology and vice versa? Is behavior and physiology, one and the same? These are all questions facing the scientific community and will require researchers from different fields to come to consensus on this issue.

After having established the fact that thermal comfort is a function of the physical system consisting of human body and thermal environment, the next step is to find a suitable thermodynamic quantity that would represent this physical system. In this study, the entropy generation $\left(S_{\text {gen }}\right)$ was discovered to be a significant thermodynamic quantity that combines both physiological and environmental variables. The concept of entropy has been introduced in human physiology by Aoki [3, 4] with the help of experimental data. According to Aoki, the entropy concept is as important as the concept of energy from the thermodynamic standpoint. All systems including the human thermal system obey 
the second law of thermodynamics. Entropy is a measure of disorder or chaos in any system in the universe. The entropy generation provides a global measure of violent motions and reactions occurring in nature. Hence, the entropy generation in the human thermal system shows the extent of activeness of (1) heat flows and (2) motions and reactions of substances within the body as a whole [3, 4]. Therefore, the entropy generation is a significant eco-physiological quantity that characterizes the combined human-thermal environment system from thermodynamic and holistic viewpoints. Thus, entropy generation $\left(S_{\text {gen }}, \mathrm{W} / \mathrm{K}\right)$ is expressed mathematically as follows:

$$
S_{\text {gen }}=f\{\text { Physiological responses, Thermal environmental variables }\}
$$

Using the expression for entropy generation, thermal comfort is redefined in terms of entropy generation using the following postulate II:

Postulate II. The entropy generation in a combined human-thermal environmental system represents the disorder or chaotic mechanisms occurring at all levels of organization. This physical disorder or chaos influences the condition of human mind stated in postulate I and thus could be used to infer thermal comfort perceived by the humans.

Using postulate II and substituting eqn (4) in eqn (3), we get the expression for thermal comfort, which is:

$$
\text { Thermal comfort }=H \times f\left\{\text { entropy generation }\left(S_{\text {gen }}\right)\right\}
$$

In order to calculate entropy generation, one needs to identify the physical variables that are categorized as follows:

1. Thermal environmental variables

Thermal resistance of the clothing $\left(I_{\mathrm{cl}}\right.$, clo)

Air temperature $\left(T_{\text {air }}, \mathrm{K}\right)$

Relative humidity (RH, \%)

Activity level or metabolic heat generation $\left(M, \mathrm{~W} / \mathrm{m}^{2}\right)$

2. Physiological responses

Skin temperature $\left(T_{\text {skin }}, \mathrm{K}\right)$

Core temperature $\left(T_{\text {core }}, \mathrm{K}\right)$

Convective heat loss from the skin surface $\left(E_{\mathrm{CNV}}, \mathrm{W}\right)$

Radiative heat loss from the skin surface $\left(E_{\mathrm{RAD}}, \mathrm{W}\right)$

Evaporative heat loss from the skin surface $\left(E_{\mathrm{EVAP}}, \mathrm{W}\right)$

Convective heat loss due to respiration $\left(E_{\mathrm{CNV} \_\mathrm{RES}}, \mathrm{W}\right)$

Evaporative heat loss due to respiration $\left(E_{\text {EVAP_RES }}, \mathrm{W}\right)$

The entropy generation term is derived from the modified expression of the second law of thermodynamics developed by Nicolis and Prigogine [12]. However, entropy generation $\left(S_{\text {gen }}, \mathrm{W} / \mathrm{K}\right)$ is found to be a function of both environmental variables and human thermal responses [5], i.e.

$$
S_{\text {gen }}=f\left\{T_{\text {skin }}, T_{\text {core }}, E_{\mathrm{CNV}}, E_{\mathrm{RAD}}, E_{\mathrm{EVAP}}, E_{\mathrm{CNV} \_ \text {RES }}, E_{\mathrm{EVAP} \_\mathrm{RES}}, M, I_{\mathrm{cl}}, T_{\text {air }}, \mathrm{RH}\right\}
$$


If an optimal comfort level with respect to entropy generation is established then OTCI is defined under postulate III as follows:

Postulate III. The percentage deviation in the value of entropy generation from the comfort or equilibrium condition provides a quantitative measure of the level of satisfaction expressed by the occupant's mind with the thermal environment and is termed as objective thermal comfort index (OTCI) expressed in percent.

OTCI is mathematically expressed as

$$
\text { OTCI }=H \times\left[1.0-\left(S_{\text {gen }}\right)_{\text {act }} /\left(\mathrm{S}_{\text {gen }}\right)_{\mathrm{com}}\right] \times 100
$$

where the subscripts 'act' and 'com' stand for actual and comfort values, respectively.

OTCI provides a measurement standard that takes into account both environmental variables and human thermal responses. The mathematical and thermodynamic details pertaining to the development of OTCI are clearly presented in Section 2.3.

2.3 Derivation of entropy generation term $\left(S_{\text {gen }}\right)$

Living organisms including human beings belong to the class of open systems. This is due to the fact that they have to continuously exchange energy and matter with the surroundings in order to maintain homeostasis, which is essential for the existence and support of life. Nicolis and Prigogine [12] developed an extended version of the second law of thermodynamics applicable to both closed and open systems.

Consider the entropy change $\mathrm{d} S$ during a time interval $\mathrm{d} t$. The entropy change $\mathrm{d} S$ is decomposed into a sum of two contributions expressed as:

$$
\mathrm{d} S=\mathrm{d} S_{\text {flow }}+\mathrm{d} S_{\text {gen }}
$$

In the present study, eqn (8) is modified as shown by Aoki $[3,4]$ as

$$
\Delta S=S_{\text {flow }}+S_{\text {gen }}
$$

where $\Delta S$ is the total entropy change, $S_{\text {flow }}$ is the net entropy flow, and $S_{\text {gen }}$ is the entropy generation.

From eqn (2), the entropy generation in the human body is given by

$$
\begin{aligned}
S_{\text {gen }} & =\Delta S-S_{\text {flow }} \\
\Delta S & =\Delta Q / T_{\text {core }}
\end{aligned}
$$

where $\Delta Q$ is the total heat generation (W) in the body

$$
S_{\text {flow }}=S_{\text {in }}-\left(S_{\text {out }}+S_{\mathrm{CNV}}+S_{\mathrm{EVAP}}+S_{\mathrm{RAD}}+S_{\mathrm{CNV} \_ \text {RES }}+S_{\text {EVAP_RES }}\right)
$$

The terms $S_{\text {in }}$ and $S_{\text {out }}$ on the right-hand side of eqn (11b) are given as follows:

$$
\begin{aligned}
S_{\text {in }} & =2.05 \sigma\left(T_{\text {air }}\right)^{3} \\
S_{\text {out }} & =2.05 \sigma\left(T_{\text {skin }}\right)^{3}
\end{aligned}
$$


where $\sigma$ is the Stefan-Boltzmann constant $=5.67 \times 10^{-8} \mathrm{~W} \mathrm{~m}^{-2} \mathrm{~K}^{-4}$. The other terms are:

$$
\begin{aligned}
S_{\mathrm{CNV}} & =E_{\mathrm{CNV}} / T_{\text {skin }} \\
S_{\mathrm{RAD}} & =E_{\mathrm{RAD}} / T_{\text {skin }} \\
S_{\mathrm{EVAP}} & =E_{\mathrm{EVAP}} / T_{\text {core }} \\
S_{\mathrm{CNV} \_ \text {RES }} & =E_{\mathrm{CNV} \_ \text {RES }} / T_{\text {skin }} \\
S_{\text {EVAP_RES }} & =E_{\text {EVAP_RES }} / T_{\text {core }}
\end{aligned}
$$

Equations (11a)-(13e) are substituted back into eqn (10) to obtain the entropy generation for both actual and comfort conditions. The entropy generation $\left[\left(S_{\text {gen }}\right)_{\text {com }}\right]$ is calculated at the equilibrium or comfort condition at $50 \% \mathrm{RH}$ and $75^{\circ} \mathrm{F}$ air temperature for a nude body. Thus, any percentage deviation from the equilibrium or comfort entropy generation value indicates the amount of deviation in thermal comfort from ideal conditions as indicated in OTCI equation (eqn (7)).

\section{RESULTS AND DISCUSSION}

OTCI was tested using simulation data obtained from the well established and validated computer model [6-9]. The continuous monitoring of thermal comfort level in both comfortable and extreme environmental conditions is demonstrated. The OTCI correlated well with the subjective PMV values obtained through regression from a large-scale experimental study.

\subsection{Thermal comfort level monitoring in comfortable environmental conditions}

The computer simulations were conducted for a period of one hour for a clothed human in comfortable environmental conditions. The effects of varying air temperature for different relative humidity values on human thermal comfort level are examined. Figure 2 shows the human thermal response in terms of OTCI to different air temperatures ranging from $286 \mathrm{~K}$ to $308 \mathrm{~K}$ for a given relative humidity of 50\%, sedentary activity $\left(M=58 \mathrm{~W} / \mathrm{m}^{2}\right)$, and medium clothing ensemble $\left(I_{\mathrm{cl}}=0.69 \mathrm{clo}\right)$.

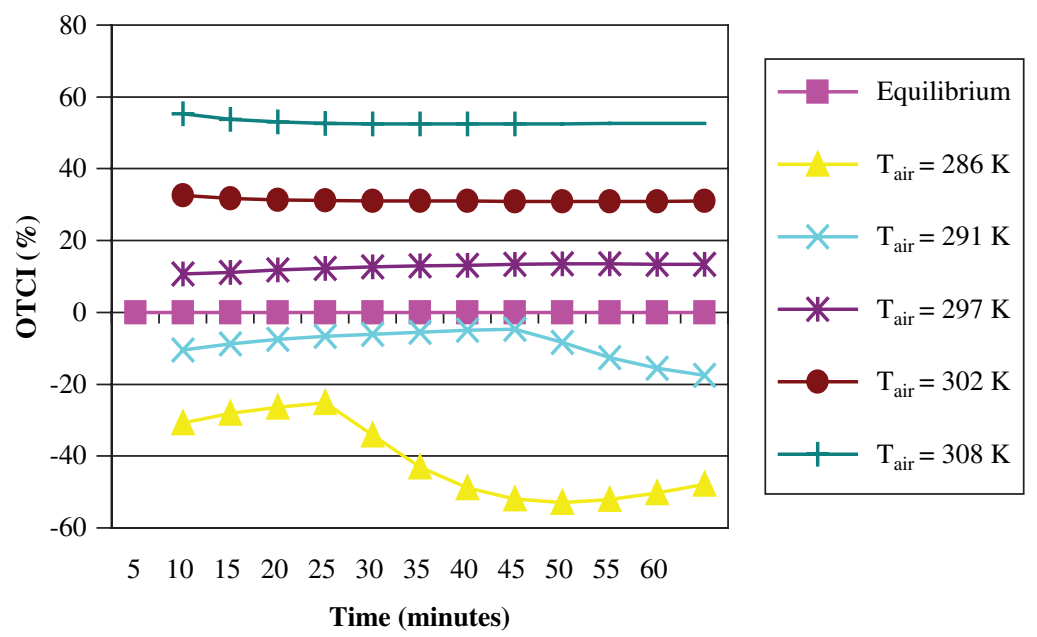

Figure 2: Effect of air temperatures on thermal comfort level for 50\% RH. 
In comfortable conditions, it is observed from Fig. 2 that there is a significant deviation in thermal comfort from the equilibrium condition. Cool air temperature $(286 \mathrm{~K})$ produced a deviation in the negative direction, starting from approximately $-30 \%$ to about $-47 \%$ towards the end of the 60th minute. As the air temperature is increased to $291 \mathrm{~K}$, the deviation from the equilibrium condition reduces starting from about $-10 \%$ to $-18 \%$. As the air temperature continues to increase, thermal discomfort shifts from the equilibrium in the positive direction. It is concluded that warmer air temperatures produce deviations in positive direction, while the cooler air temperatures produce shifts in the negative direction from the thermal equilibrium of the body. Also, it is noticed that OTCI indicates a sudden change in thermal stress level at the 25th and 45th minute for air temperatures of $286 \mathrm{~K}$ and $291 \mathrm{~K}$, respectively. This is mainly attributed to the onset of heat production due to shivering, which contributes to the increase of entropy production.

It can be observed from Fig. 3 that the relative humidity does not have significant effect on the human perception of thermal comfort for a given air temperature of $294.26 \mathrm{~K}$, sedentary activity, and a medium clothing of thermal resistance value 0.69 clo. It is concluded that the range of $30 \%-70 \%$ $\mathrm{RH}$ lies in the comfort zone of the ASHRAE comfort chart [1]. Thus, it can be noted from Fig. 3 that there is negligible thermal discomfort in this range which is indicated by OTCI quantitatively approximately ranging from a value of $-1 \%$ to a value of $+4 \%$.

\subsection{Thermal comfort level monitoring in extreme hot and cold environments}

Simulations were conducted for a period of $1 \mathrm{~h}$ for a sedentary clothed human in extreme ambient conditions. The effects of extreme cold $(273 \mathrm{~K})$ and hot $(316 \mathrm{~K})$ air temperatures for $10 \% \mathrm{RH}$ are examined as shown in Fig. 4. In case of extreme environmental conditions, it is observed from Fig. 4 that for a $10 \% \mathrm{RH}$, the deviations from the thermal equilibrium are large indicating thermal discomfort of extreme cold and hot for $273 \mathrm{~K}$ and $316 \mathrm{~K}$ air temperatures, respectively. Further, it is noticed that there is an onset of shivering at the 10th minute for the $273 \mathrm{~K}$ air temperature.

Figure 5 demonstrates the fact that the level of thermal comfort experienced by the occupant at $90 \% \mathrm{RH}$ and $273 \mathrm{~K}$ and $316 \mathrm{~K}$ is same as that experienced at $10 \% \mathrm{RH}$. It can be concluded that the relative humidity does not have an impact on thermal comfort even at very cold and hot temperatures. This could be attributed to the fact that the human computer model used in the study does not have enough sensitivity to changes in relative humidity. However, it would be interesting to explore the effects of relative humidity on human perception of comfort in an experimental study.

\section{CONCLUSIONS}

The results demonstrate the application of the second law of thermodynamics to quantitatively assess and evaluate the human-thermal environmental interaction. The present study focused mainly on

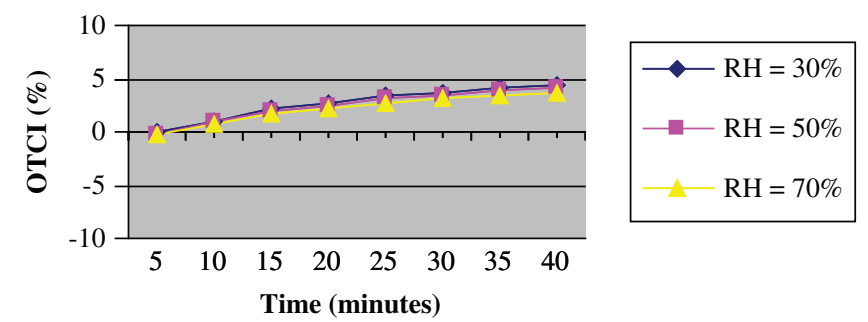

Figure 3: Effect of relative humidity on OTCI at 294.26 K air temperatures. 


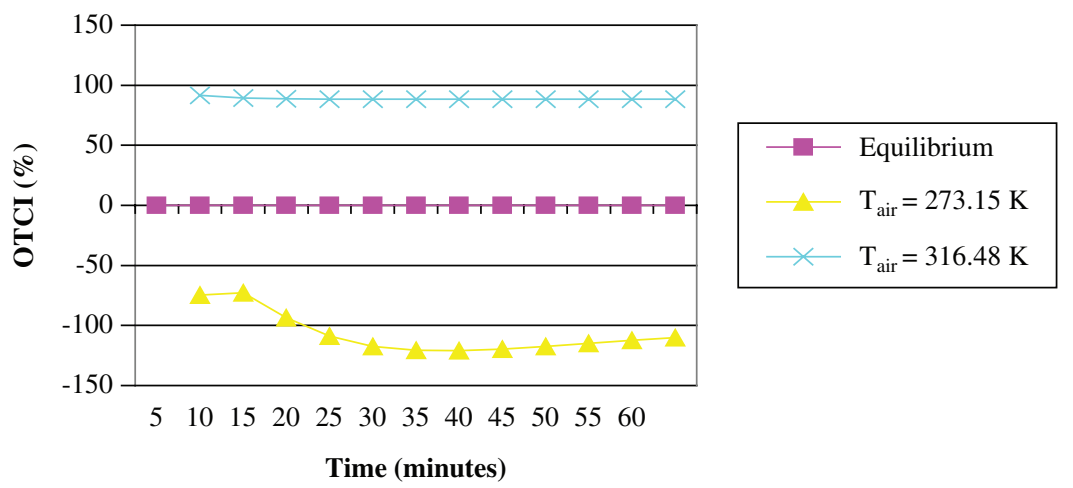

Figure 4: Thermal comfort in extreme air temperatures for $10 \% \mathrm{RH}$.

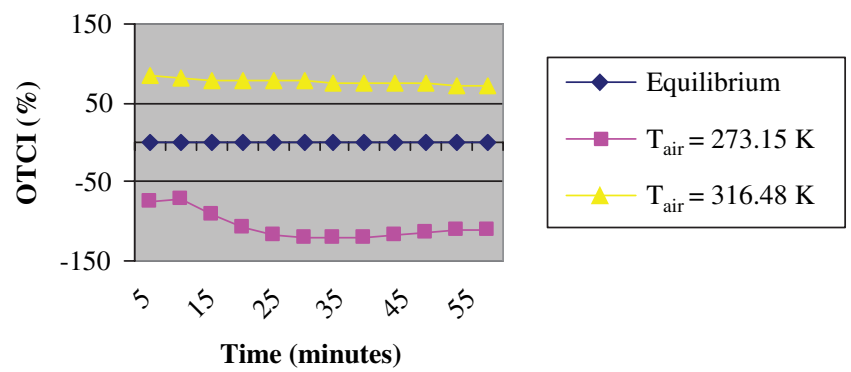

Figure 5: Thermal comfort in extreme air temperatures for 90\% RH.

data from simulations and OTCI to infer human perception of thermal comfort and thereby provide a quantitative means to design human-centered energy-efficient environmental control systems. This study, mainly based on computer simulations, was done mainly to overcome the cost of experimental studies and lack of resources on the part of the authors. In order to implement the OTCI in an operational environment, an experimental study needs to be conducted. With the availability of advanced non-invasive and non-contact sensors to measure human thermal physiological responses and the environmental variables, it would be possible to design and conduct an experimental study in the future. The results from such a study would greatly benefit the scientific community to explore the relationship between thermal environments and human physiology from thermodynamic and energetic perspectives leading to human-centered eco-sustainable solutions.

\section{REFERENCES}

[1] ASHRAE, Thermal comfort (Chapter 8). ASHRAE Handbook of Fundamentals, SI Edition, ASHRAE, Inc.: Atlanta, 2005.

[2] Talty, J.T., Industrial Hygiene Engineering, 2nd edn, Noyes Data Corporation: New Jersey, pp. 326-369, 1988.

[3] Aoki, I., Effects of exercise and chills on entropy production in human body, Journal of Theoretical Biology, 145(1), pp. 421-428, 1990.

[4] Aoki, I., Entropy flow and production in human body in basal conditions. Journal of Theoretical Biology, 141(6), pp. 11-21, 1989. 
[5] Boregowda, S.C., Thermodynamic Modelling and Analysis of Human Stress Responses, Doctoral Dissertation, Old Dominion University, Norfolk, Virginia, USA, 1998.

[6] Smith, C.E., A Transient, Three-Dimensional Model of the Human Thermal System, Doctoral Dissertation, Kansas State University, Manhattan, Kansas, USA, May 1991.

[7] Fu, G., A Transient, Three-Dimensional Mathematical Thermal Model for Clothed Human, Doctoral Dissertation, Kansas State University, Manhattan, Kansas, May 1995.

[8] Boregowda, S.C., \& Tiwari, S.N., Numerical investigation of thermal comfort using a finite element human thermal model. AIAA Paper No. 2002-2877, Presented at the 8th Joint AIAA/ASME Thermophysics and Heat Transfer Conference, St. Louis, MO, June 2002.

[9] McGuffin, R., Burk, R., Huizenga, C., Hui, Z., Vlahinos, A., \& Fu, G., Human thermal comfort model and manikin. Society of Automotive Engineers Paper No. 2002-01-1955, 2002 SAE Future Car Congress, Arlington VA, 4 June 2002, http://www.aes.nu/publications/ 2002-01-1955.pdf.

[10] Fechner, G., Elements of Psychophysics, Vol. I, Holt, Rinehart and Winston, Inc.: New York, 1966.

[11] Fanger, P.O., Thermal Comfort, 2nd edn, McGraw-Hill Book Company: New York, 1970.

[12] Nicolis, G. \& Prigogine, I., Self-Organization in Nonequilibrium Systems, John Wiley \& Sons: New York, 1977. 